\title{
PERFIL SOCIODEMOGRÁFICO E CLÍNICO DE INDIVÍDUOS DIAGNOSTICADOS COM HANSENÍASE
}

\author{
SOCIODEMOGRAPHIC AND CLINICAL PROFILE OF LEPROSY \\ PATIENTS
}

\author{
Rafaela Rolim de Oliveira ${ }^{1}$ \\ Ankilma do Nascimento Andrade Feitosa ${ }^{2}$ \\ Maria Berenice Gomes Nascimento ${ }^{3}$ \\ Myrelle Kelly Pereira Januário ${ }^{4}$ \\ Jessika Lopes Figueiredo Pereira Batista ${ }^{5}$ \\ Talina Carla da Silva ${ }^{6}$
}

RESUMO: Objetivo: Caracterizar perfil sociodemográfico e clínico dos indivíduos diagnosticados com hanseníase. Método: Trata-se de um estudo de campo, descritivo, exploratório, com abordagem quantitativa. A pesquisa ocorreu no município de Cajazeiras, nas Unidades Básicas de Saúde do município em questão. A amostra foi composta por 15 registros de pacientes diagnosticados com hanseníase no período de 2018 a agosto de 2019, que se adequavam aos critérios de inclusão e exclusão. Os dados da pesquisa foram obtidos através dos prontuários dos pacientes com hanseníase que foram notificados nas unidades de saúde, os quais foram trabalhados de forma descritiva simples. A pesquisa seguiu respeitando os aspectos éticos presentes na Resolução n 466, de 12 de dezembro de 2012 do Conselho Nacional de Saúde. Resultados: a pesquisa evidenciou que o público mais acometido pela hanseníase é o sexo masculino, haja vista sua maior vulnerabilidade ao adoecimento e cuidado reduzido com a saúde, com estado cívil casado, faixa etária acima de 60 anos de idade, com ensino fundamental incompleto. No que se refere aos dados clínicos dos pacientes, a forma clínica mais

\footnotetext{
${ }^{1}$ Enfermeira pela Faculdade Santa Maria. Professora Substituta da Universidade Federal de Campina Grande.

${ }_{2}^{2}$ Doutora em Ciências da Saúde pela Faculdade de Medicina do ABC. Professora da Faculdade Santa Maria.

${ }^{3}$ Mestre em Saúde Coletiva pela Universidade Católica dos Santos. Professora da Universidade Federal de Campina Grande.

${ }^{4}$ Graduada em Enfermagem pela Universidade Federal de Campina Grande.

5 Mestre em Saúde Pública pela Universidade Estadual da Paraíba. Professora Substituta da Universidade Federal de Campina Grande.

6 Doutora em Ciências da Saúde pela Escola de Enfermagem da Universidade de São Paulo. Docente da Faculdade Santa Maria e Coordenadora da Pós-Graduação da Faculdade de Santa Maria de Cajazeiras.
} 
predominante foi a dimorfa da classificação operacional multibacilar, a qual é responsável pela manutenção da cadeia de transmissibilidade da doença na sociedade, e com maior acometimento neurológico o que aumenta as chances do desenvolvimento de incapacidades físicas. No que diz respeito ao grau de incapacidade física com maior representatividade foi o grau I, o qual é definido a partir do comprometimento da sensibilidade e da força muscular sem deformidades visíveis. Conclusão: Dessa forma, a detecção precoce de casos novos, principalmente no público mais vulnerável, se torna imprescindível não só para o controle da doença, como também para evitar a evolução da patologia para formas clínicas mais graves e com mais alto risco de danos ao estado físico do indivíduo doente, que pode resultar em sequelas que afetam significativamente a sua qualidade de vida.

Descritores: Análise de vulnerabilidade. Epidemiologia. Hanseníase. Nervos periféricos.

ABSTRACT: Objective: To characterize the sociodemographic and clinical profile of individuals diagnosed with leprosy. Method: This is a descriptive, exploratory field study with a quantitative approach. The research took place in the city of Cajazeiras, in the Basic Health Units of the municipality in question. The sample consisted of 15 records of patients diagnosed with leprosy from 2018 to August 2019, which met the inclusion and exclusion criteria. The research data were obtained from the medical records of leprosy patients who were notified in the health units, which were worked in a simple descriptive manner. The research continued respecting the ethical aspects present in Resolution No. 466 of December 12, 2012 of the National Health Council. Results: The research showed that the public most affected by leprosy is male, given their greater vulnerability to disease and reduced health care, with married civil status, age above 60 years, with incomplete elementary school. Regarding the clinical data of the patients, the most predominant clinical form was the dimorphic multibacillary operational classification, which is responsible for maintaining the disease transmissibility chain in society, and with greater neurological involvement, which increases the chances of developing of physical disabilities. Regarding the degree of physical disability with greater representativeness was grade I, which is defined from the impairment of sensitivity and muscle strength without visible deformities. Conclusion: Thus, early detection of new cases, especially in the most vulnerable public, is essential not only for disease control, but also to prevent the evolution of the disease to more severe clinical forms and with a higher risk of damage to the disease. physical condition of the sick individual, which can result in sequelae that significantly affect their quality of life.

Keywords: Vulnerability analysis. Epidemiology. Leprosy. Peripheral nerves. 


\section{INTRODUÇÃO}

A hanseníase, patologia que acomete pessoas no mundo todo há mais de três mil anos, é uma doença crônica, infectocontagiosa, cujo agente etiológico é o Mycobacterium leprae, um bacilo álcool-ácido resistente, o qual atinge os nervos periféricos, bem como os nervos e células superficiais da pele, resultando no acometimento dermatoneurológico da doença, o que confere a mesma um alto potencial incapacitante, especialmente quando diagnosticada e tratada tardiamente (BRASIL, 2017).

No que diz respeito à sua transmissibilidade, a mesma ocorre de forma direta, por via respiratória, através do contato com uma pessoa doente, sem tratamento, especialmente Multibacilar (MB), com carga elevada de bacilos, que eliminam o agente para o meio exterior infectando outras pessoas suscetíveis ao adoecimento (BRASIL, 2010).

A estatística global da hanseníase, no ano de 2012, revelou que menos de 20 países mencionaram mais de 1000 casos novos, mostrando que essa enfermidade está aos poucos se resumindo a um pequeno número de países. Entre estes, o Brasil aparece ocupando o segundo lugar, com 33.303 novos casos notificados no decorrer do ano em questão, ficando só atrás da Índia, com 134.752 novos casos no mesmo ano. Vale ressaltar ainda que, o Brasil apareceu como o país das Américas com a maior quantidade de crianças diagnosticadas também em 2012, com 6,7\%, mostrando uma exposição precoce a essa infecção (WHO, 2013).

Quando comparado esses dados de 2012 com os dados registrados no ano de 2016, no Brasil, onde foram notificados 25.218 novos casos, finalizando um número de detecção de 12,2/100 mil habitantes, é notório que houve uma redução significativa, no entanto algumas regiões como Nordeste, Norte e Centro-Oeste são consideradas como zonas endêmicas (BRASIL, 2015; BRASIL, 2017). Esse decréscimo do número de pessoas com hanseníase é decorrente do aumento das ações para combater a doença (BRASIL, 2014). 
Apesar da redução no número de casos noticiados nos últimos anos, a patologia em questão ainda se configura como um problema de saúde pública, especialmente nas regiões mais carentes do Brasil, que quando não diagnosticada e tratada precocemente evolui, podendo resultar em comprometimento físico devido ao acometimento neurológico, mantendo ainda a transmissibilidade da doença que pode atingir pessoas de qualquer sexo ou faixa etária. Dessa forma, o diagnóstico e o tratamento precoce dos casos de hanseníase compreendem medidas eficazes para se prevenir as incapacidades físicas decorrentes da doença (BRITO et al., 2014b).

Tais incapacidades e deformidades físicas resultantes da hanseníase podem acarretar problemas de ordem psicossocial, como por exemplo, redução da capacidade de trabalho, limitação da vida social, isolamento pela própria sociedade e até mesmo família, devido ao forte estigma ainda presente e consequentemente depressão (BITTENCUOR et al., 2006).

É de suma importância, ressaltar também nesse contexto o papel que a Estratégia Saúde da Família (ESF) apresenta, a equipe de saúde que faz parte de um processo coletivo de trabalho, atua diretamente nas ações de controle da hanseníase seja individualmente com a pessoa doente, sua família ou comunidade. Os profissionais atuam na perspectiva da prevenção da doença, busca e diagnóstico, tratamento e seguimento dos casos, prevenção e tratamento das incapacidades físicas ou deformidades irreversíveis decorrentes da doença (RODRIGUES et al., 2015).

Considerando o contexto supracitado o profissional de enfermagem membro da equipe apresenta papel de grande relevância no acompanhamento dos indivíduo com diagnóstico de hanseníase, seja na realização do tratamento, objetivando uma melhor adesão ao mesmo, seja na prevenção de incapacidades físicas, realizando além do seguimento terapêutico, a detecção precoce de novos casos, mediante busca ativa na comunidade, especialmente em áreas endêmicas, e através da avaliação de comunicantes de casos confirmados da referida doença (RIBEIRO; CASTILLO; OLIVEIRA, 2017).

Com isso, objetivou-se caracterizar perfil sociodemográfico e clínico dos indivíduos diagnosticados com hanseníase. 


\section{METODOLOGIA}

Trata-se de um estudo de campo, descritivo, exploratório, com abordagem quantitativa. Segundo Prodanov e Freitas (2013), o estudo descritivo apresenta como finalidade descrever determinada população ou fenômeno, ou até mesmo o estabelecimento de relações entre variáveis, onde o investigador descreve os fatos que são observados sem intervir nos mesmos, fazendo-se o uso de técnicas específicas padronizadas para a coleta dos dados, como o questionário, entrevista e observação sistemática.

De acordo com Traldi e Dias (2011), o estudo exploratório tem por finalidade aprofundar o conhecimento a cerca de um fenômeno, visando explorar sua complexidade, identificando os fatores associados ao seu desenvolvimento como também as várias maneiras através das quais se manifesta. Sendo um estudo mais desenvolvido onde se tem conhecimento acumulado e sistematizado, insatisfatório ou até mesmo inexistente.

A pesquisa ocorreu no município de Cajazeiras, o qual está localizado no Sertão Paraibano, a 477 quilômetros de sua capital João Pessoa. Esta cidade faz parte da 4⿳亠丷a Macrorregião de Saúde e 9ª Gerência Regional de Saúde da Paraíba. O local de execução do estudo propriamente dito foi nas Unidades Básicas de Saúde (UBS) do município em questão, o qual apresenta um total de 23 unidades, das quais 18 estão localizadas na zona urbana e cinco na zona rural.

A população do estudo em tela foi composta por 55 casos notificados de hanseníase no município de Cajazeiras-PB, de acordo com dados do Sistema de Informação de Agravos de Notificação (SINAN) do período de 2018 à agosto de 2019, enquanto que a amostra foi composta por 15 registros, que estavam dentro dos critérios de inclusão e exclusão.

Foram incluídos na pesquisa os casos de hanseníase notificados no período de 2018 a agosto de 2019, residentes no município, com idade igual ou superior a 18 anos, sendo excluídos os casos registrados nas UBS pertencentes a zona rural, 
devido ao deslocamento, bem como os casos com dados incompletos nos prontuários.

Após submissão, através da Plataforma Brasil, ao Comitê de Ética em Pesquisa (CEP) em Seres Humanos do Centro de Formação de Professores da Universidade Federal de Campina Grande, os dados da pesquisa foram obtidos através dos prontuários dos pacientes que foram notificados nas UBS, a partir das informações referentes ao perfil sociodemográfico e clínico dos casos registrados, com base em um roteiro estruturado para coleta.

Os dados sociodemográficos foram trabalhados utilizando a estatística descritiva simples, os quais foram armazenados em uma planilha eletrônica do Microsoft Office Excel 2003. Para tabulação e confecção das tabelas foi utilizado o Microsoft Word (2010), sendo todos discutidos à luz da literatura pertinente ao tema.

A pesquisa seguiu respeitando os aspectos éticos presentes na Resolução $n^{\circ}$ 466, de 12 de dezembro de 2012 do Conselho Nacional de Saúde (CNS), após parecer favorável de número 3.588.922, atendendo todas normas de pesquisas com seres humanos, incorporando os quatro referenciais básicos da biótica: autonomia, não maleficência, beneficência e justiça.

\section{RESULTADOS}

Foram analisados 15 registros de casos de hanseníase notificados no município de Cajazeiras-PB, no período estudado, os quais são apresentados nas tabelas abaixo, referentes ao perfil sociodemográfico, como também perfil clínico. 
Tabela 1: Perfil sociodemográfico dos casos notificados de hanseníase. CajazeirasPB.

\begin{tabular}{|l|c|c|}
\hline VARIÁVEIS & $\boldsymbol{F}$ & $\%$ \\
\hline SEXO & & \\
\hline MASCULINO & 9 & 60 \\
\hline FEMININO & 6 & 40 \\
\hline Total & 15 & 100 \\
\hline
\end{tabular}

\section{ESTADO CIVIL}

SOLTEIRO (a)

$4 \quad 26,6$

CASADO (a)

$8 \quad 53,3$

OUTROS

3

Total

15

\section{FAIXA ETÁRIA}

30 a 39

40 a 49

50 a 60

Acima de 60

Total

\begin{tabular}{|c|c|}
\hline 2 & 13,3 \\
\hline 4 & 26,6 \\
\hline 3 & 20 \\
\hline 5 & 33,3 \\
\hline 15 & 100 \\
\hline
\end{tabular}

ESCOLARIDADE

FUNDAMENTAL INCOMPLETO

FUNDAMENTAL COMPLETO

MÉDIO COMPLETO

SUPERIOR

Total

Fonte: Dados da Pesquisa - 2019.

Observa-se que nos dados apresentados na tabela 1 é possível identificar uma predominância do sexo masculino com relação ao feminino, com estado cívil casado, faixa etária acima de 60 anos, com fundamental incompleto.
53,3

6,6

26,6

13,3

100 
Tabela 2: Perfil clínico dos casos notificados de hanseníase. Cajazeiras-PB.

\begin{tabular}{|c|c|c|}
\hline VARIÁVEIS & $\boldsymbol{F}$ & $\%$ \\
\hline \multicolumn{3}{|l|}{ FORMA CLIINICA } \\
\hline INDETERMINADA (PB) & 3 & 20 \\
\hline TUBERCULÓIDE (PB) & 3 & 20 \\
\hline DIFORMA (MB) & 8 & 53,3 \\
\hline VIRCHOVIANA (MB) & 1 & 6,6 \\
\hline Total & 15 & 100 \\
\hline \multicolumn{3}{|c|}{ GRAU DE INCAPACIDADE FÍSICA } \\
\hline 0 & 0 & 0 \\
\hline 1 & 13 & 86,6 \\
\hline 2 & 2 & 13,3 \\
\hline Total & 15 & 100 \\
\hline
\end{tabular}

Fonte: Dados da Pesquisa - 2019.

A tabela 2 evidencia que dentre os casos notificados a forma clínica mais predominante foi a dimorfa, da classificação operacional Multibacilar (MB), com grau de incapacidade física1.

\section{DISCUSSÃO}

Os dados encontrados com o estudo evidenciam uma predominância da hanseníase na população masculina, e a caracterização da patologia por sexo permite indicar diferenças de acesso em termos da capacidade de alcance do programa e da capacidade da população em utilizar os serviços de saúde, e o cuidado com a mesma. Além disso, é possível identificar variações na carga da doença entre os grupos populacionais, verificando assim, as lacunas ainda existentes no que se refere às práticas de saúde destinadas a cada público.

Quando se discute a prevalência da doença em relação ao sexo, observa-se que tanto no que diz respeito à ocorrência da hanseníase, como a própria forma operacional do tipo MB, são mais prevalentes nos homens, o que na grande maioria 
das vezes pode ser explicado pela maior vulnerabilidade deste público à infecção, seja por uma maior exposição ao agente etiológico, seja pelo cuidado reduzido com a sua saúde, resistência em buscar o serviço de saúde, o que afeta significativamente o tempo do diagnóstico, contribuindo para o desenvolvimento das incapacidades físicas (MOURA et al., 2008).

Vale ressaltar que, quanto mais tardiamente for o diagnóstico e instituição do tratamento maiores são as chances da evolução da doença para formas mais graves e incapacitantes. E apartir dos achados com a pesquisa em tela, tendo em vista a predominância da doença em questão, no público masculino, torna-se ainda mais preocupante, quando considerado a maior resistência deste grupo específico em procurar os serviços de saúde precocemente, em aderir até mesmo a terapêutica e adotar as práticas de autocuidado diárias.

As diferenças nas taxas de detecção entre homens e mulheres apontam para a necessidade de estratégias diferenciadas, que contemplem a diversidade do panorama da hanseníase no país, considerando especialmente o sexo mais acometido. Apesar dos avanços ao longo dos últimos anos, é necessário garantir a melhoria dos serviços de saúde, principalmente no que tange à atenção básica, com ampliação da descentralização das ações como estratégia fundamental para redução da carga da doença, especialmente nas áreas com maior concentração de casos da patologia. Com isso, é de grande relevância priorizar e intensifcar ações de educação em saúde, busca ativa de novos casos, vigilância de contatos, qualificação do diagnóstico, principamente em tempo hábil, prevenção e tratamento de incapacidades físicas, com enfoque diferenciado para homens e mulheres, considerando o público mais vulnerável ao adoecimento (CABRAL et al., 2016).

O estudo também evidenciou que, em relação ao estado civil há uma diferença significativa com relação ao número de solteiros, casados e outros, onde foi possível observar que houve predominância de pacientes com estado civil casado, com um quantitativo de oito casos $(53,3 \%)$, este fato pode contribuir de forma positiva seja no enfretamento da doença, seja na realização do tratamento de forma correta, considerando o suporte familiar, em especial do cônjugue.

Os estudos demontram que os pacientes que são casados ou tem alguma pessoa ao seu lado, tem demosntrando mais cuidado com a sua saúde, aumentando 
a procura pelo serviço de saúde , principamente para o serviço porta de entrada preferencial, Atenção Básica, haja vista suas parceiras terem maior preocupação com o cuidado da sua familia, fazendo com que os homens se sintam mais seguros para o enfrentamento de qualquer patologia que possa surgir (LOPES; RANGEL, 2014).

No que diz respeito à faixa etária, observou-se uma predominância da doença no público estudado, em pessoas com idade mais avançada, ou seja, na população com mais de 60 anos de idade, seguida da popualção em idade economicamente ativa.

Dessa forma, observa-se que tal fato pode ser justificado quando considerado o sistema imunológico da pessoa idosaa, o qual encontra-se comprometido em decorrência do próprio envelhecimento, além de considerar que a hanseníase é uma doença de evolução crônica, e que pode apresentar um longo peíodo de incubação.

Vale ressaltar ainda, que o indivíduo idoso pode apresentar um acometimento ainda mais severo da doença, desenvolver formas mais graves, tendo em vista que a mesma promove acometimento dos nervos periféricos resultando em diminuição da sensibilidade tátil e de força muscular, o que pode afetar ainda mais a qualidade de vida da pessoa idosa, e resultar em incapacidades físicas, o que pode debilitar ainda mais o indivíduo acometido pela doença (ARAUJO, 2003).

O envelhecimento, desencadeia modificações graduais e inevitáveis; e no decorrer do mesmo, alterações podem ocorrer, tornando o indivíduo mais vulnerável e suscetível à várias doenças e agravos, especialmente patologias infectocontagiosas como a própria hanseníase, e esta pode se manifestar ainda de forma mais grave no público em questão, o que pode resultar em sequelas muitas vezes limitantes que afetam a qualidade de vida do idoso acometido (MEDEIROS et al., 2015).

No que concerne à escolaridade a pesquisa evideciou uma predominância da doença em indivíduos com ensino fundamental incompleto, o que pode contribuir significativamente para esta realidade o baixo nível de instrução desse público, o que pode afetar tanto no que diz respeito ao entendimento da doença, como também na adoção de práticas de prevenção de doenças e agravos.

No que se refere aos dados clínicos, oberservou-se que a maioria dos 
registros evidenciam diagnóstico da forma transmissível da hanseníase, a qual é responsável pela manutenção da doença na sociedade, especialmente para seus contatos familiares. A forma clínica mais predominante foi a dimorfa, da classificação operacional MB, que além do alto potencial de transmissibilidade, acarreta maior comprometimento neurológico e consequentemente comprometimento físico, que quando não tratada precocemente de forma correta, resulta em incapacidades físicas, que pode ser definida a partir da avaliação do grau de incapaciade física, o qual é determinado no início do diagnóstico e final do tratamento, pelo profissional de saúde responsável pela avaliação da pessoa doente.

Nos pacientes com diagnóstico de hanseníase, é fundamental a avaliação da incapacidade física, mediante avaliação de sensibilidade, força, especialmente na região de olhos, mãos e pés, e esta avaliação faz parte do tratamento, sendo realizada durante seguimento do mesmo, haja vista o potencial da doença em comprometer funções de grande importância na vida do indivíduo (NOGUEIRA et al., 2017).

A hanseníase, quando não diagnosticada e tratada oportunamente e corretamente com a poliquimioterapia preconizada pela Organização Mundial de Saúde (PQT/OMS), pode evoluir para formas mais graves e incapacitantes, resultando em incapacidades físicas muitas vezes irreversíveis, acarretando limitações na vida da pessoa atingida pela doença, comprometendo além do seu estado físico, o socioeconômico e psicológico do indivíduo (BRITO et al., 2014a).

Dessa forma, de acordo com Nogueira et al. (2017), o grau de incapacidade física é definido mediante avaliação neurológica, no momento do diagnóstico e após término da terapia medicamentosa, do paciente por parte de um profissional de saúde que realiza atendimento ao doente, por meio de impresso específico padronizado pelo Ministério da Saúde, o qual contempla avaliação dos olhos, mãos e pés, no que diz respeito às alterações visuais, queixas referidas pela pessoa doente, e avaliação da função neural, a qual compreende, sensibilidade protetora ocular, palmar e plantar, força muscular e palpação de nervos periféricos atingidos. Essa avaliação é expressa em valores que variam de zero a dois, e consiste em ferramenta fundamental para que seja traçado o plano de cuidado mais adequados para cada paciente. 
No estudo, observou-se que a maioria dos pacientes possui ou possuíam no momento do diagnóstico o grau de incapacidade física I, representado por um total de $86,6 \%$, o que significa que estes possuem/possuíam a perda de sensibilidade ou de força muscular, a qual pode afetar olhos, mãos e pés.

Essas complicações podem ser responsáveis por sequelas permanentes ao indivíduo, pois, podem atingir os receptores dos nervosos responsáveis pela dor, visão e sensibilidade tátil, tornando-os mais susceptíveis a acidentes, queimaduras, feridas e, até mesmo, amputações, resultando em danos sociais e psíquicos que interferem na qualidade de vida da pessoa acometida (MONTEIRO et al., 2013).

\section{CONCLUSÃO}

A hanseníase, mesmo com todos os recursos disponíveis no que diz respeito ao diagnóstico, tratamento e seguimento terapêutico, ainda é considerada um desafiador problema de saúde pública, especialmente no Brasil, e quando analisado o público mais acometido pela doença, observa-se a população masculina como sendo a mais vulnerável ao adoecimento, quando considerado a maior exposição dessa população ao agente causador, cuidado reduzido com a saúde, ou até mesmo a não procura pelo serviço de saúde como medida de prevenção, o que pode estar associado à questões culturais, por exemplo.

Vale ressaltar, que além dessa maior vulnerabilidade ao adoecimento, o homem ainda pode apresentar maiores chances de um acometimento mais severo da doença, e consequentemente evolução para incapacidades físicas, haja vista a hanseníase possuir um alto potencial incapacitante devido ao comprometimento neurológico da doença. Dessa forma, quanto mais tardiamente a pessoa for diagnosticada e tratada maiores as chances de desenvolvimento de danos físicos, que podem ser irreversíveis.

Ainda no que diz respeito aos dados sociodemográficos, a doença foi mais prevalente na população com idade superior a 60 anos, o que pode estar associado ao próprio sistema imune da pessoa idosa, decorrente do processo de 
envelhecimento; e em indivíduos com baixo grau de instrução, o que pode afetar tanto na compreensão da doença, como na adoção de práticas de prevenção e autocuidado.

Quanto ao quadro clínico, observa-se um maior quantitativo de casos com a forma clínica dimorfa e grau de incapacidade física I, o que evidencia um quantitativo de pessoas com a forma transmissível da doença e de maior acometimento neurológico, que pode aumentar as chances de sequelas para pessoa atingida, as quais podem ser evitadas mediante um diagnóstico precoce e realização de tratamento em tempo oportuno, e avaliação regular destes paciente durante tratamento.

Dessa forma, a detecção precoce de casos novos se torna imprescindível não só para o controle da doença, como também para evitar a evolução da patologia para formas clínicas mais graves e com mais alto risco de danos ao estado físico do indivíduo doente, que pode resultar em sequelas que afetam significativamente a sua qualidade de vida.

\section{REFERÊNCIAS BIBLIOGRÁFICAS}

ARAÚJO, M. G. Hanseníase no brasil. Rev. Soc. Bras. Med. Trop, v. 33, n. 3, p. 373- 382, 2003.

BITTENCOURT, L. P. et al. Estigma: percepções sociais reveladas por pessoas acometidas por hanseníase. Rev. enferm. UERJ, Rio de Janeiro, v. 18, n. 2, p. 185-190, 2010.

BRASIL. Ministério da Saúde. Secretaria de Vigilância em Saúde. Departamento de Secretaria de Vigilância em Saúde, Departamento de Vigilância Epidemiológica. - 8. ed. rev. - Brasília: Ministério da Saúde, 2010.

BRASIL. Ministério da saúde. Secretaria de vigilância em saúde. Departamento de vigilância das doenças transmissíveis. Coordenação geral de hanseníase e doenças em eliminação. Informe Técnico: Campanha Nacional de Hanseníase, Verminoses, Tracomae Esquistossomose 2015. Brasília, 2015.

BRASIL. Ministério da Saúde. Secretária de Vigilância em Saúde Doenças em eliminação. Brasília: 2014.

BRASIL. Ministério da Saúde. Secretaria de Vigilância em Saúde. Departamento de Vigilância das Doenças Transmissíveis. Guia prático sobre a hanseníase. Brasília: Ministério da Saúde, 2017.

BRITO, K.K.G. et al. Práticas e limitações de clientes com hanseníase no cuidar das lesões cutâneas. Rev. enferm. UFPE on line., Recife, v. 8, n. 1, p. 7-16, 2014 a. 
BRITO , K. K. G. et al. Análise epidemiológica da hanseníase em um estado do Nordeste Brasileiro. Rev. de enferm. UFPE, Recife, Vol. 8, n. 8, p. 93-2686, 2014 b.

CABRAL, C. V. S. et al. O papel do enfermeiro na prevenção de incapacidades e deformidades no portador de hanseníase. Revista Interdisciplinar, v. 9, n. 2, p. 168-177, 2016.

LOPES, V.A.S.; RANGEL, E.M. Hanseníase e vulnerabilidade social: uma análise do perfil socioeconômico. Rev. Saú. em Deb. Rio de Janeiro, v. 38, n. 103, p. 817-829, out-dez, 2014.

MEDEIROS, A. P. S. et al. Perfil de pessoas com e sem comorbidades acometidas por reações hansênicas. Cogitare Enfermagem, v. 20, n. 2, p. 281-8, abr-jun, 2015.

MONTEIRO, L. D. et al. Incapacidades físicas em pessoas acometidas pela hanseníase no período pós-alta da poliquimioterapia em um município no Norte do Brasil, Cad. Saúde Pública, Rio de Janeiro, v. 29, n. 5, p. 909-920, mai, 2013.

NOGUEIRA, P. S. F. et al. Fatores associados à capacidade funcional de idosos com hanseníase. Rev. Bras. Enferm, Brasília, v. 70, n. 4 July-Aug, 2017.

MOURA, R. S. de et al. Sorologia da hanseníase utilizando PGL-I: revisão sistemática. Rev Soc Bras Med Trop, 41 (suplemento II), p. 11-18, 2008.

PRODANOV, C. C.; FREITAS, E. C. Metodologia do Trabalho Científico: Métodos e Técnicas da Pesquisa e do Trabalho Acadêmico. Ed.2, Novo Hamburgo: Feevale, 2013.

RIBEIRO, M. D. A.; CASTILLO, I. S.; SILVA, J. C. A.; OLIVEIRA, S. B. A visão do profissional enfermeiro sobre o tratamento da hanseníase na atenção básica. Rev. Bras. Prom. Saúd., Fortaleza. v, 30, n. 2, p. 221-228, 2017.

RODRIGUES, F.F. et al. Conhecimento e prática dos enfermeiros sobre hanseníase: ações de controle e eliminação. Rev. Bras. de Enf., v. 68, n. 2, p. 297-304, 2015.

TRALDI, M. C.; DIAS, R. Monografia passo a passo. Ed. 7, Campinas-SP: Editora Alínea, 2011.

WORLD HEALTH ORGANIZATION (WHO). Global leprosy: update on the 2012 situation. Weekly epidemiological record, Geneva. v. 35, n. 88, p. 80-365, 2013. 